\begin{tabular}{|c|l|}
\hline Title & $\begin{array}{l}\text { Phase transition behaviors of the supported DPPC bilayer investigated by sum frequency generation (SFG) vibrational } \\
\text { spectroscopy and atomic force microscopy (AFM) }\end{array}$ \\
\hline Author(s) & Wu, Heng-Liang; Tong, Y ujin; Peng, Qiling; Li, Na; Ye, Shen \\
\hline Citation & $\begin{array}{l}\text { Physical chemistry chemical physics, 18(3), 1411-1421 } \\
\text { https://doi.org/L0.1039/c5cp04960a }\end{array}$ \\
\hline Issue Date & 2016-01-21 \\
\hline Doc URL & http://hdl.handle.net/2115/64237 \\
\hline Type & article(author version) \\
\hline File Information & PCCP_revised_Highlighted.pdf \\
\hline
\end{tabular}

Instructions for use 


\title{
Phase Transition Behaviors of the Supported DPPC Bilayer Investigated by Sum Frequency Generation (SFG) and Atomic Force Microscopy (AFM)
}

\author{
Heng-Liang Wu, Yujin Tong, Qiling Peng, Na Li, and Shen Ye* \\ Catalysis Research Center, Hokkaido University, Sapporo 001-0021, Japan
}

\begin{abstract}
The phase transition behaviors of a supported bilayer of dipalmitoylphosphatidylcholine (DPPC) have been systematically evaluated by in situ sum frequency generation (SFG) vibrational spectroscopy and atomic force microscopy (AFM). By using an asymmetric bilayer composed of per-deuterated and per-protonated monolayers, i.e., DPPC-d75/ DPPC and symmetric bilayer of DPPC/DPPC, we were able to probe the molecular structural changes during the phase transition process of the lipid bilayer by SFG spectroscopy. It was found that the DPPC bilayer is sequentially melted from the top (adjacent to the solution) to bottom leaflet (adjacent to the substrate) over a wide temperature range. The conformational ordering of the supported bilayer does not decrease (even slightly increases) during the phase transition process. The conformational defects in the bilayer can be removed after the complete melting process. The phase transition enthalpy for the bottom leaflet was found to be approximately three times greater than that for the top leaflet, indicating a strong interaction of the lipids with the substrate. The present SFG and AFM observations revealed similar temperature dependent profiles. Based on these results, the temperature-induced structural changes in the supported lipid bilayer during its phase transition process are discussed in a comparison with previous studies.
\end{abstract}




\section{Introduction}

The cell membrane separates the intracellular components from the extracellular environment and plays important roles in a variety of cellular processes such as cell adhesion, ion conductivity and cell signaling. ${ }^{1-3}$ Therefore, tremendous attention has been focused on its chemical and physical properties. One of the important properties is the thermal-induced phase transition of the cell membrane. Usually, the membrane actively functionalizes in its liquid phase. It is known that the phase transition for the membrane occurs around the melting temperature $\left(T_{m}\right)$ of lipids. ${ }^{4-5}$ The phase transition process can significantly affect many of the physical and chemical properties of the cell membranes, such as conformation ordering, ${ }^{6-9}$ rate of the flip-flop process, ${ }^{10}$ molecular density ${ }^{11}$ as well as the enzymatic activity. ${ }^{12-15}$

The phase transition behaviors of the cell membranes have been investigated using different model systems, such as the lipid vesicle, lipid monolayer, and supported lipid bilayer. Differential scanning calorimetry (DSC) measurements showed that the phase transition of the large multilamellar vesicles (LMVs) of lipids occurs at the $T_{m}$ with a very narrow temperature transition range $\left(<1^{\circ} \mathrm{C}\right) .5,16-18$ The temperature range for the phase transition becomes wider by downsizing the vesicles. The phase transition for small unilamellar vesicles (SUVs) with diameters of 15-50 $\mathrm{nm}$ could occur in a temperature range wider than $10^{\circ} \mathrm{C}$ due to its high surface curvature. ${ }^{18-20}$ By using Fourier transform infrared (FTIR) absorption spectroscopy, Mantsch et al. reported that the hydrocarbon chains of the LMVs of dipalmitoylphosphatidylcholine (DPPC) become disordered during the phase transition around $41^{\circ} \mathrm{C} .8^{8-9}$

Recently, the supported lipid bilayers are getting more attention as a model system to study the fundamental processes of the biological membrane.21-22 The supported lipid bilayer can provide a wide and flat area that can be studied by other techniques such as 
DSC, ${ }^{23}$ infrared and Raman spectroscopy, ${ }^{24-25}$ sum frequency generation spectroscopy (SFG), ${ }^{26-29}$ and atomic force microscopy (AFM). ${ }^{30-36}$ Bain and coworkers investigated the phase transition process of a supported lipid bilayer prepared by the fusion of SUVs onto a fused silica surface by Raman spectroscopy in a total internal reflection geometry. ${ }^{24}$ They reported that the chain disordering and the tilt angle of the lipids increase with temperature. They also found that the phase transition for the supported lipid bilayers occurs over a wide temperature range $\left(\sim 10^{\circ} \mathrm{C}\right)$, commencing at the calorimetric phase transition temperature.

The morphological changes in the supported lipid bilayers prepared from the LMV suspension during the phase transition have been evaluated by AFM. In the early stage, a one-step transition behavior at $T_{m}$, i.e., the gel phase directly melts into the liquid phase, was observed. ${ }^{37-39}$ Leonenko et al. later reported a two-step transition behavior of a supported DPPC bilayer on the mica surface (Fig. 1a). ${ }^{30}$ In this model, the gel phase changes into the "liquid disorder phase" above the $T_{m}$, while an intermediate state of the "liquid order phase" is present at the $T_{m}$. Similar morphological changes were also observed by other groups, but a somewhat different model was proposed to explain the phenomena. ${ }^{33-35}$ This later proposed model (Fig. 1b) suggested that the top leaflet adjacent to the solution first melts into the liquid phase near the $T_{m}$ (the intermediate state of the "partial melting of the bilayer" was called the "crack phase", Fig. 1b), and then the bottom leaflet starts to melt with the increasing temperature. The full melting process occurs in the temperature range of ca. $10^{\circ} \mathrm{C}$. The two-stage model (Fig. 1b) agrees with the previous DSC results obtained for a supported lipid bilayer on a mica surface showing split phase transition peaks around the $T_{m}{ }^{23}$ The phase transition behaviors seem to be significantly affected by the interaction of the lipids with the substrates. On the other hand, Seeger et al. reported that the decoupled phase transition of the supported lipid bilayer is also influenced by their surrounding environments, such as the ionic strength, $\mathrm{pH}$ as well as the lipid types. ${ }^{40-41}$ 
(a)

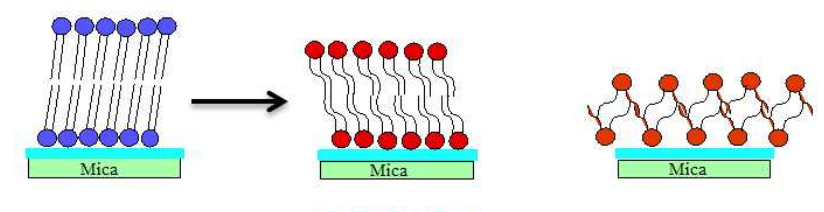

(b)

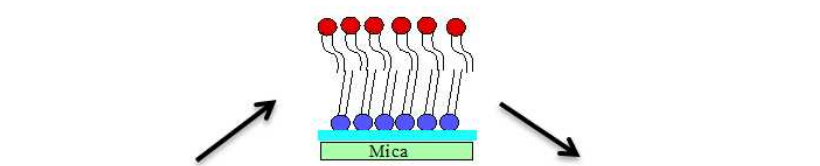

(c)

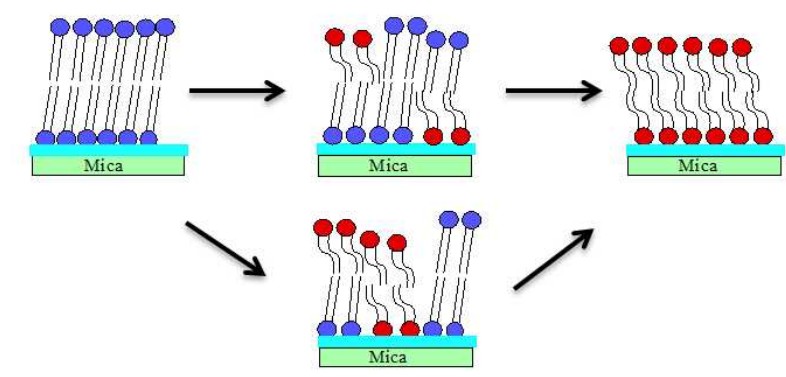

below $T_{m}$

at $T_{m}$

above $T_{m}$

Figure 1. Several models previously proposed for the phase transition process of the support lipid bilayer from the gel to liquid phase, illustrating (a) domain disordering model, (b) "crack" phase model, (c) domain dislocation model, and (d) domain size disparity model. See text for details.

Recently, by utilizing the advantage of the high surface sensitivity and selectivity of sum frequency generation (SFG) vibrational spectroscopy, ${ }^{42-47}$ Conboy and coworkers reported an SFG study on the structural changes of the supported lipid bilayers on a fused quartz surface during the phase transition. ${ }^{26-28}$ They found that the SFG intensity of the methyl group of the phospholipid's alkyl chains shows a maximum near the $T_{m}$ with a wide temperature response $\left(\sim 10^{\circ} \mathrm{C}\right)$. Thus, they suggested that SFG spectroscopy could be used to probe the $T_{m}$ of the supported lipid bilayer. They proposed a domain dislocation model (Fig. 1c) and domain size disparity model (Fig. 1d) to explain the temperature dependent SFG behaviors for the supported lipid bilayer. At a temperature near the $T_{m}$, the first model suggests that the same amount of lipids are melted in both the top and bottom leaflets but at different positions, while the latter model proposes that the different sizes of the gel and liquid domains are present in the two leaflets. Both models could generate a large asymmetry in the lipid bilayer at the $T_{m}$, thus contributing to the SFG signals. These models 
are different from those proposed from the AFM observation mentioned above although the latter one (Fig. 1b) can provide the largest asymmetry between the top and bottom leaflets in the bilayer and thus can also explain the SFG signal maximum at the $T_{m} \cdot{ }^{33-35}$ It is hard to simply judge which model is correct or not since these AFM and SFG measurements are carried out under somewhat different sample preparation conditions in different groups. For example, most of the supported lipid bilayers for the AFM observations were prepared by the vesicle fusion method, ${ }^{33-35}$ while those for the SFG observations were prepared by the Langmuir-Blodgett (LB) method. ${ }^{26-29}$ One may argue that the difference in the model could be attributed to the different experimental conditions. To clarify the discrepancy in these models, a careful study is highly desired by using these two probing techniques under similar experimental conditions.

In the present study, both the broad-band SFG and AFM approaches were used to investigate the phase transition behaviors of a DPPC bilayer under the similar conditions. The broad-band SFG system allows us to continuously record the SFG spectra in a wide region $\left(\sim 250 \mathrm{~cm}^{-1}\right)$ and thus we are able to get more information than previous SFG observations with higher time resolution. Based on these systematic observations by the two techniques, we are able to obtain a comprehensive understanding of the phase transition behaviors of the supported lipid bilayers.

\section{Experimental}

\section{Sample preparation}

The conditions for the preparation of the lipid bilayers are the same as in our previous studies. ${ }^{48-49}$ The per-protonated DPPC and per-deuterated DPPC- $\mathrm{d}_{75}$ were purchased from Sigma-Aldrich and Avanti Polar Lipids, Inc., respectively. The lipid monolayers were first prepared by spreading a $1 \mathrm{mg} / \mathrm{ml}$ chloroform solution of DPPC on the pure water subphase in an LB trough (FSD-500, USI). The upper and lower leaflets for the supported 
lipid bilayer were successively deposited onto a substrate surface at a pressure of $30 \mathrm{mN} / \mathrm{m}$ (corresponding to a gel phase with an average area of $0.50 \mathrm{~nm}^{2}$ per molecule) by two vertical dipping processes. In addition to the symmetric bilayer of the DPPC/DPPC (in the present top/bottom bilayer notation, top and bottom leaflets represent the layer adjacent to solution and substrate, respectively), an asymmetric bilayer composed of per-deuterated and per-protonated DPPC molecules, i.e., DPPC-d $75 /$ DPPC or DPPC/DPPC-d 75 , was also prepared in the SFG experiments. Based on this design, one expects to independently probe the structural changes in each leaflet. ${ }^{46}$

The substrate for the SFG measurement was a flat surface of hemicylindrical fused quartz. To enable the SFG measurement for the vibrational modes in the lower frequency region (such as $\mathrm{C}-\mathrm{D}$ stretching and $\mathrm{C}=\mathrm{O}$ stretching), a $\mathrm{CaF}_{2}$ substrate was also used. To make the $\mathrm{CaF}_{2}$ surface hydrophilic, the bottom surface of the $\mathrm{CaF}_{2}$ surface was coated with a $\mathrm{SiO}_{2}$ thin-film (ca. 20-nm thickness) by the sol-gel method. The substrate for the AFM observations was freshly-cleaved mica. Special care was taken for the bilayer transfer to the AFM or SFG cells under water. The AFM experiments were carried out in pure water or a $0.1 \mathrm{M}$ Tris-buffer solution ( $\mathrm{pH}$ 8.9) containing $5 \mathrm{mM} \mathrm{CaCl} 2$ in a homemade AFM cell, ${ }^{36}$ while that of the SFG measurements were performed in pure water. As will be described later, no difference in the AFM images was observed between those in pure water and Tris-buffer solution in the present study.

\section{$\underline{\text { AFM measurements }}$}

An Agilent 5500 (Agilent Technologies, USA) was used in the tapping mode at the typical scan rate of $1 \mathrm{~Hz}$. The silicon nitride $\left(\mathrm{Si}_{3} \mathrm{~N}_{4}\right)$ cantilever (Olympus, RC800PSA-1) has a spring constant of $0.76 \mathrm{~N} / \mathrm{m}$, a resonance frequency of $\sim 10 \mathrm{kHz}$, and tip radius of $20 \mathrm{~nm}$. All the height mode AFM images were acquired under in situ conditions in solution. Imaging was conducted with a small force to limit the destruction of the lipid bilayer. Typically, it took $4 \mathrm{~min}$ to record one AFM image $\left(256 \times 256\right.$ pixels per image). ${ }^{36}$ 
The temperature control during the AFM observation was realized using the Peltier sample stage (Agilent Technologies, USA). The sample stage was connected to a temperature controller, Model 332 (Lake Shore Cryotronics, Westerville, OH, USA), providing a heating current to the sample stage. The typical heating rate was $5.0 \mathrm{~K} / \mathrm{min}$. A thermocouple (type K) was installed in the AFM cell to monitor the temperature though a digital multimeter (Agilent 34401A) and its temperature output was recorded by a personal computer through a USB interface (1 point / 2 seconds).

The temperature in the AFM cell was controlled between room temperature (ca. $24^{\circ} \mathrm{C}$ ) and ca. $55^{\circ} \mathrm{C}$ with a temperature step of $0.5^{\circ} \mathrm{C}$. After the temperature reached the desired value, the AFM observation was paused for at least $20 \mathrm{~min}$ to ensure equilibrium at that temperature and then the cantilever re-approached the bilayer surface to start the next AFM observation. Thus, the AFM images were usually acquired in different areas as the temperature was changed. At least two sequential AFM images with the same ratio of different phases were obtained before the next temperature step. Nevertheless, since the bilayer surface is believed to be uniform in a region of $\mu \mathrm{m}$ order, the morphologies obtained on the different positions of the sample are generally the same on average. For example, the ratios of the different phases in the bilayer have been characterized on different areas of the bilayer, and no difference could be found under the same conditions when the imaging area is greater than $1 \mu \mathrm{m}^{2}$. The solution was refreshed every 2 hours by adding a pre-warmed solution at the same temperature.

The AFM images were analyzed by the Scanning Probe Image Processor SPIP 5.1.2 (Image Metrology A/S, Denmark) and the time mark associated with each image was taken as the end of the scan.

\section{$\underline{\text { SFG measurements }}$}

A broad-band SFG system ${ }^{50-51}$ consisted of a Ti:sapphire oscillator and a regenerative amplifier pumped by a Nd:YLF laser generating a $2.2 \mathrm{~mJ}$ output at $800 \mathrm{~nm}$ with a $120 \mathrm{fs}_{\mathrm{S}}$ 
pulse duration and a repetition rate of $1 \mathrm{kHz}$. Half of the output was used to pump an optical parametric amplifier system to generate an IR wavelength tunable from 2.5 to $10 \mu \mathrm{m}$ with a spectral width of ca. $250 \mathrm{~cm}^{-1}$. The remaining output from the amplifier was sent to a homemade spectral shaper to generate a narrow-band pulse $\left(\sim 10 \mathrm{~cm}^{-1}\right)$ at $800 \mathrm{~nm}$ for improving the spectral resolution.

Incident angles of the visible $(800 \mathrm{~nm})$ and infrared beams were $70^{\circ}$ and $55^{\circ}$, respectively. In the present experiment, the central wavelength of IR beam was fixed at 3.50 $\mu \mathrm{m}$ for C-H stretching $\left(2800 \sim 3000 \mathrm{~cm}^{-1}\right)$ for the per-protonated DPPC and $4.50 \mu \mathrm{m}$ for the C-D stretching $\left(2000 \sim 2300 \mathrm{~cm}^{-1}\right)$ for the per-deuterated DPPC- $\mathrm{d}_{75}$. The SFG spectra were recorded with the polarization combination of both the $s s p$ (s-SFG, $s$-visible and $p$-IR) and ppp.

All the SFG measurements were carried out under in situ conditions in contact with an aqueous solution. The temperature of the SFG cell was controlled by a water jacket attached through a chiller (NCB-3200, EYELA) between 5 to $75^{\circ} \mathrm{C}$. The typical heating/cooling rate was $0.22^{\circ} \mathrm{C} / \mathrm{min}$, which allows a sufficient temperature resolution with a continuous temperature dependent SFG measurement (1 spectrum/min). No difference was found for the lower heating/cooling rate. A thermocouple installed in the cell was used to monitor the temperature of the supported lipid bilayer.

\section{Results and Discussion}

Phase transition behavior of the supported DPPC bilayers studied by SFG

The in situ SFG measurement was carried out between $12^{\circ} \mathrm{C}$ and $55^{\circ} \mathrm{C}$. Figure 2 shows a series of the temperature dependent ssp-polarized SFG spectra $\left(2800-3000 \mathrm{~cm}^{-1}\right)$ for an asymmetric DPPC-d75/DPPC bilayer deposited on a $\mathrm{CaF}_{2}$ surface during the heating 
process. Due to the asymmetric structures of the bilayer design, we were able to selectively probe the bottom leaflet, namely that adjacent to the substrate, i.e., DPPC layer in the C-H stretching region. Before heating, the as-prepared asymmetric bilayer showed two major peaks at 2878 and $2938 \mathrm{~cm}^{-1} .^{29}$ The two peaks can be assigned to the symmetric C-H stretching $\left(\mathrm{CH}_{3}, \mathrm{ss}\right)$ and Fermi resonance $\left(\mathrm{CH}_{3}, \mathrm{FR}\right)$ between the $\mathrm{C}-\mathrm{H}$ symmetric and overtone of the $\mathrm{C}-\mathrm{H}$ bending of the terminal methyl $\left(\mathrm{CH}_{3}\right)$ group of the DPPC molecules in the bottom leaflet of the asymmetric bilayers. A small shoulder is also observed at $2850 \mathrm{~cm}^{-}$ 1, which can be assigned to the symmetric $\mathrm{C}-\mathrm{H}$ stretching mode $\left(\mathrm{CH}_{2, \mathrm{ss}}\right)$ of the methylene $\left(\mathrm{CH}_{2}\right)$ group. This indicates that a relatively ordered DPPC monolayer with a small amount of gauche defects was constructed as the bottom leaflet in the bilayer. The SFG peak intensity is directly proportional to the square of the population of the DPPC molecules in the bottom leaflet, or critically speaking, the population difference of DPPC between the bottom and top leaflets (the as-prepared asymmetric bilayer only has DPPC in the bottom leaflet). In addition to the population difference, the spectral intensity and shape can also be affected by the structural difference between the two leaflets in the supported bilayer.

Figure $2 \mathrm{~b}$ shows the intensity changes of $\mathrm{CH}_{3}$,ss as a function of the temperature (black solid circles, Fig. 2b). As the bilayer is heated, the spectral intensity seems to be constant until ca. $30^{\circ} \mathrm{C}$ (denoted as stage I). The spectral intensity then gradually decreases to a local minimum around $40^{\circ} \mathrm{C}$ (denoted as stage II) and further increases to a local maximum around $47^{\circ} \mathrm{C}$ (denoted as stage III) and finally drop to low signal level at higher temperatures. No new peaks and peak position shift are observed during this heating process. 
During stage I, the bilayer seems to keep the same structure with heating. As the temperature is raised to stage II, the rate of the flip-flop movement of the lipid molecules between the top and bottom leaflets mostly increases. The flip-flop of lipids in the bilayer always occurs and its rate significantly accelerates with temperature, especially when it is close to the $T_{m}$. Part of the DPPC in the bottom leaflet and DPPC- $\mathrm{d}_{75}$ in the top leaflet will flop up and flip down to the opposite leaflet, respectively. As a result, the population difference of the DPPC molecules between the bottom and top leaflets becomes smaller and the SFG intensity decreases. As the temperature is further raised to stage III, the phase transition of the DPPC in the supported bilayer is expected to take place. The SFG signal increase in stage III should not be attributed to the flip-flop process which only makes the bilayer more symmetric and thus produced a weaker SFG signal. An additional structural change is expected to occur with the phase transition. As described in the Introduction, Conboy et al. proposed two models of domain dislocation (Fig. 1c) and domain size disparity (Fig. 1d) to explain the observed behaviors, especially the SFG spectral maximum during the phase transition process. ${ }^{27}$ Although these models could predict a local maximum of the SFG spectral intensity during the phase transition temperature by considering the structural differences in the top and bottom leaflets, these are not the only
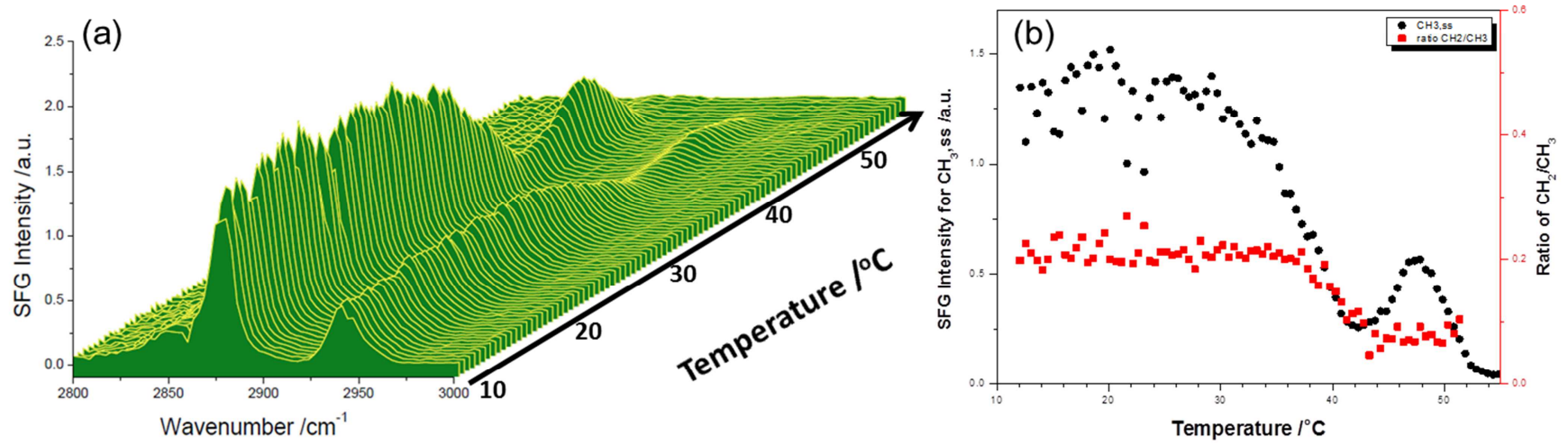

Figure 2. (a) The ssp-polarized SFG spectra DPPC- $d_{75} / D P P C$ asymmetric bilayer deposited on a CaF ${ }_{2}$ surface in contact with water as the temperature increases from 12 to $55^{\circ} \mathrm{C}$. (b) Temperature dependence of SFG intensity of $\mathrm{CH}_{3}, \mathrm{Ss}$ and ratio of $\mathrm{CH}_{2} / \mathrm{CH}_{3}$. See text for details. 
structures that can give the present temperature dependent SFG behaviors. Other models, for example, the crack phase model in Fig. $1 \mathrm{~b}$, can also explain the temperature dependent behaviors. Additional evidence is necessary to clarify the real structural changes. Furthermore, as already mentioned, these models (Figs. 1c and 1d) are contradicted by the recent in situ AFM observations of a similar system.

It should also be mentioned that here although the temperature dependent profiles are similar to those previously reported by Conboy et al. for an asymmetric bilayer of DPPC on a fused quartz surface, the local minimum and maximum of the SFG signals for the asymmetric bilayer on the $\mathrm{CaF}_{2}$ substrate surface (Fig. 2) are approximately $+5^{\circ} \mathrm{C}$ higher than Conboy's results. ${ }^{26-28}$ As will be discussed later in this paper, the interaction between the lipids and substrate significantly affect the phase transition enthalpy of the bilayer; one of the possible reasons for the difference is expected to be a stronger interaction between the DPPC and $\mathrm{CaF}_{2}$ substrate. This may also partially relate to the differences of the heat conductivities of the different substrates. Nevertheless, as will be discussed later, the phase transition mechanism does not change with the substrate. In order to compare this with previous studies, a fused quartz substrate was adopted for other SFG observations only in the C-H stretching region of the study.

Although only a very small spectral change was observed in stage I, we found that the tilt angle of the alkyl chain of DPPC slightly changes with temperature before the phase transition. The $p p p$-polarized SFG measurement demonstrated that the tilt angle of the alkyl chain increases with temperature from $0( \pm 3)$ degrees $\left(10^{\circ} \mathrm{C}\right)$ to $12( \pm 3)$ degrees $\left(30^{\circ} \mathrm{C}\right)$ for the DPPC both in the top and bottom leaflets of the supported bilayer (see Fig. S1 in Supporting Information). Similar changes in the tilt angles of the supported lipid bilayer have previously been reported by Bain and coworkers using in situ ATR-Raman observations. ${ }^{24}$ Unfortunately, as the temperature increases, the flip-flop rate is significantly 
accelerated, so the asymmetric features of the bilayer quickly disappear, thus the present spectral analysis becomes difficult.

On the other hand, it was found that the spectral intensity for $\mathrm{CH}_{2}$ changes in a similar way as that for $\mathrm{CH}_{3}$ with temperature. To investigate the structural disordering during the heating process, the ratios between the peak amplitude of the $\mathrm{CH}_{2}$ and $\mathrm{CH}_{3}$ peaks at different temperatures were determined from the in situ SFG spectra (red solid blocks, Fig. $2 b)$. This ratio is widely employed to characterize the conformational disordering of organic molecules with long alkyl chains. ${ }^{50-53}$ As shown in Fig. 2b, the ratio remains constant during stage I and starts to decrease in stage II to a lower value in stage III. This indicates that the conformation ordering does not decrease (even slightly increases) during the phase transition process. This result is different from those previous DSC and Raman observations which reported that the bilayer became more disordered during the phase transition. The present SFG observation is expected to be more reliable due to SFG's unique and high responses to the conformation disordering such as gauche defects. In fact, the SFG peak for the $\mathrm{CH}_{2}$ peak completely disappeared during the cooling process from the liquid phase to gel phase even when the temperature was reduced to room temperature (Fig. S2, see Supporting Information). This implies that the conformational defects in the bilayer are removed after the full melting process. On the other hand, our SFG results suggest that the phase transition behavior of the supported DPPC bilayer was not fully reversible during heating and cooling process (see Fig. S2).

The asymmetric lipid bilayer is a nice approach to investigate the structural changes in each individual leaflet in the bilayer by SFG spectroscopy. Due to the existence of the flip-flop process, the population of the per-deuterated or per-protonated DPPC in each leaflet also changes with temperature and time, which makes the quantitative analyses difficult. To solve this problem, a symmetric bilayer of DPPC/DPPC deposited on a fused 
quartz substrate was further investigated to quantitatively characterize the phase transition. Due to the symmetric structure of DPPC/DPPC, the flip-flop process will not affect the SFG spectral intensity and it is easier to figure out the origins for the spectral changes with temperature.

Figure 3a shows a series of ssp-polarized SFG spectra $\left(2800-3000 \mathrm{~cm}^{-1}\right)$ for the symmetric DPPC/DPPC bilayer on a fused quartz surface during the heating process. Two peaks are observed at 2878 and $2938 \mathrm{~cm}^{-1}$, which can be attributed to $\mathrm{CH}_{3}, \mathrm{ss}$ and $\mathrm{CH}_{3}, \mathrm{FR}$ of DPPC. No peak attributed to $\mathrm{CH}_{2}$ is observed. The symmetric bilayer (Fig. 3a) shows much lower spectral intensity in comparison to the asymmetric bilayer (Fig. 2a). This is reasonable since the terminal $\mathrm{CH}_{3}$ groups of the alkyl chains in the symmetric bilayer locate face-to-face and thus their SFG contributions are cancelled out by the destructive interference. The SFG peaks are still weakly observed since the top and bottom leaflets in the DPPC/DPPC bilayer are not exactly identical in their chemical environment. A small difference in molecular density between the top and bottom leaflets may also contribute to the SFG spectrum for the symmetric bilayer.

Figure $3 \mathrm{~b}$ shows the peak intensity of $\mathrm{CH}_{3}$,ss as a function of temperature. The peak
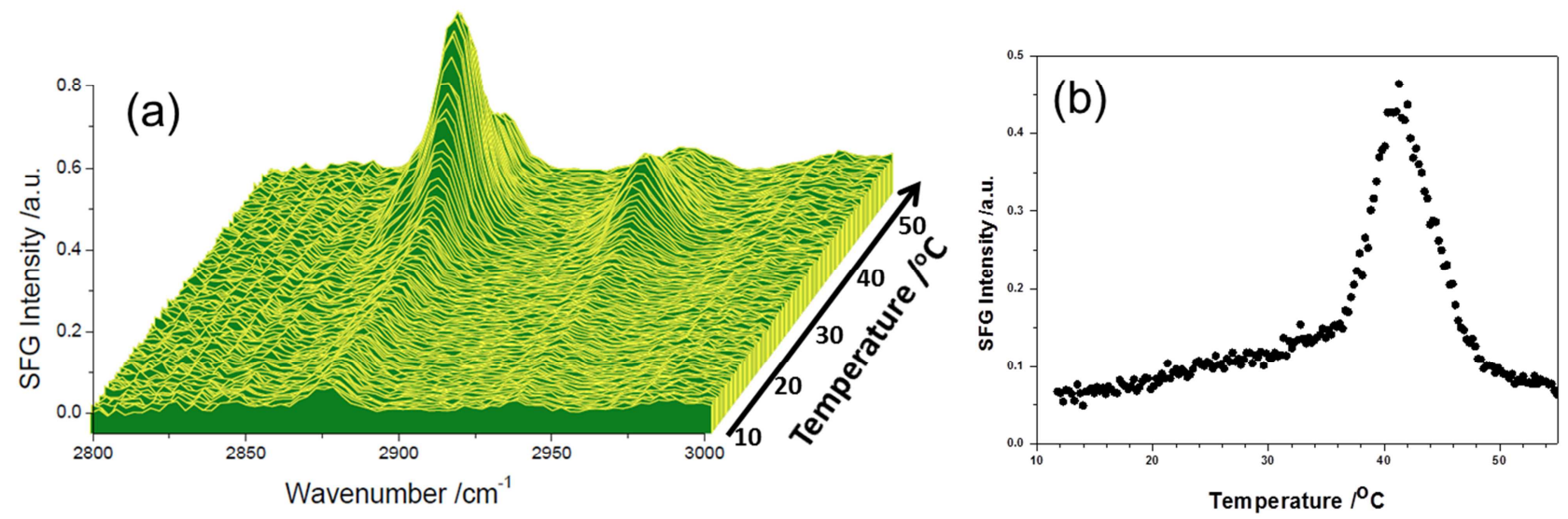

Figure 3. (a) in situ SFG spectra of the symmetric DPPC/DPPC bilayer on a fused quartz surface in contact with water as the temperature increases from 10 to $55^{\circ} \mathrm{C}$. The heating rate was $0.22^{\circ} \mathrm{C} / \mathrm{min}$ while the SFG spectra were recorded at a rate of 1 spectrum per minute. (b) Normalized SFG peak intensity at 2878 $\mathrm{cm}^{-1}$ as a function of temperature for the bilayer upon heating (black trace). 
intensity for $\mathrm{CH}_{3}$,ss slightly increases with temperature and starts to quickly increase when the temperature is higher than $35^{\circ} \mathrm{C}$. A local maximum for the SFG peak intensity of $\mathrm{CH}_{3}$,ss is observed around $42^{\circ} \mathrm{C}$ (Fig. 3b). The present temperature profile of the symmetric bilayer of DPPC on a fused quartz surface is exactly identical to that previously reported by Conboy et al. ${ }^{27-28}$ No temperature shift in the local maximum of the SFG signal was observed when a fused quartz substrate was used. These spectral changes should be undoubtedly associated with the phase transition process of the supported DPPC bilayer. As discussed in Fig. 2, the temperature dependent SFG spectral changes directly reflect the symmetric difference between the top and bottom leaflets of the supported bilayer. The local maximum in the SFG intensity profile indicates that the largest structure asymmetry between the two leaflets of the symmetric bilayer is generated at this temperature. In fact, the previously presented interpretation for stage III in Fig. 2 can be used to explain the present observation for the symmetric bilayer. However, we are still unable to conclude which model (Fig.1 a-d) describes what really happened to the supported bilayer at the $T_{m}$. To fully answer this question, the phase transition behaviors of the lipid bilayers will be further characterized by in situ AFM.

On the other hand, no peak attributed to the $\mathrm{CH}_{2}$ group was observed during the heating process (Fig. 3a). This confirms again that the conformational ordering of the bilayer is fully maintained during the phase transition process as that in the asymmetric bilayer (Fig. 2). It is interesting to note that the phase transition process occurs within a relatively wide temperature range. Similar phenomena were also found for the asymmetric DPPC bilayer (Fig. 2). This is in agreement with previous studies on the supported bilayer, ${ }^{24,27-28,33-35}$ but different from a sharp phase transition process for lipid vesicles in solution $\left(<1^{\circ} \mathrm{C}\right),{ }^{5,16-18}$ implying the unique feature for the phase transition on the supported lipid bilayer system. 


\section{Phase transition behavior of supported DPPC bilayers studied by AFM}

Figure 4 shows height mode AFM images $\left(2 \times 2 \mu \mathrm{m}^{2}\right)$ of a supported DPPC bilayer deposited at $30 \mathrm{mN} / \mathrm{m}$ during a heating process from $25^{\circ} \mathrm{C}$ (gel phase) to $50^{\circ} \mathrm{C}$ (liquid phase). The cross section profiles for these AFM images are given in Fig. S3. The AFM image of a supported DPPC bilayer at $25^{\circ} \mathrm{C}$ (Fig. 4a) shows a wide flat terrace in the gel phase (denoted as the domain (i), same as below). A number of small holes with depths of $\sim 5 \mathrm{~nm}$, corresponding to the height of a well-defined supported DPPC bilayer, are observed and can be regarded as defects in the DPPC bilayer in the gel phase. ${ }^{36,54}$ The domain (i) dominates the supported bilayer surface when the temperature is low.

As the temperature is raised to $33^{\circ} \mathrm{C}$, some new domain structures with lower heights (domain (ii), Fig. 4b) appear on the extended bilayer surface in the gel phase (domain (i)). Domain (ii) is approximately $0.6 \pm 0.2 \mathrm{~nm}$ lower than domain (i), and becomes wider at elevated temperature. The thickness of domain (ii) is ca. $4.4 \mathrm{~nm}$. Similar features have also been previously reported by other groups. ${ }^{34-35}$

The AFM image at $40^{\circ} \mathrm{C}$ (Fig. 4c) shows that most of the supported DPPC bilayer surface is dominated by domain (ii) with only a small amount of domain (i). Furthermore, a very small amount of the lower domain structures starts to appear at this temperature (denoted as domain (iii), Fig. 4c). In other words, three domains co-exist at this temperature. As the temperature is slightly raised to $41.5^{\circ} \mathrm{C}$, domain (i) completely disappears and only domain (ii) and a very small amount of domain (iii) exist (Fig. 4d). This indicates that the gel phase of domain (i) melts at this temperature $\left(41 \pm 1^{\circ} \mathrm{C}\right)$. The repeated AFM observations indicated that the phase transition process from the gel phase to the new phase takes place over the wide temperature range from $33 \pm 1^{\circ} \mathrm{C}$ to $41 \pm 1^{\circ} \mathrm{C}$.

As temperature is further raised, domain (iii) starts to increase $\left(42.5^{\circ} \mathrm{C}\right.$, Fig. $\left.4 \mathrm{e}\right)$. 
Domain (iii) expands with temperature. As shown in Fig, $4 \mathrm{f}\left(49^{\circ} \mathrm{C}\right)$, domain (iii) dominates the bilayer surface. Domain (ii) completely disappears and only domain (iii) is present, indicating the full melting of the bilayer into domain (iii).

Domain (iii) is approximately $0.6 \pm 0.2 \mathrm{~nm}$ lower in height than domain (ii), and can be regarded as a fully melted lipid bilayer (i.e., liquid phase). The height difference is similar to that observed between domains (i) and (ii), implying that these changes may be associated with the structural changes in each individual leaflet. In other words, domains (ii) and (iii) correspond to the locally melting phase on the top and bottom leaflet, respectively. Thus the height of the fully melted DPPC bilayer (i.e., domain (iii)) is $5.0-0.6-0.6=3.8 \mathrm{~nm}$. This value is in good agreement with a previous result by X-ray diffraction. ${ }^{55}$ The phase change for domain (ii) to (iii) occurs in the temperature range of $7 \sim 8{ }^{\circ} \mathrm{C}$.

Feng et al. previously reported similar AFM images of the supported lipid bilayers with the existence of three stages during the phase transition process and attributed it to the
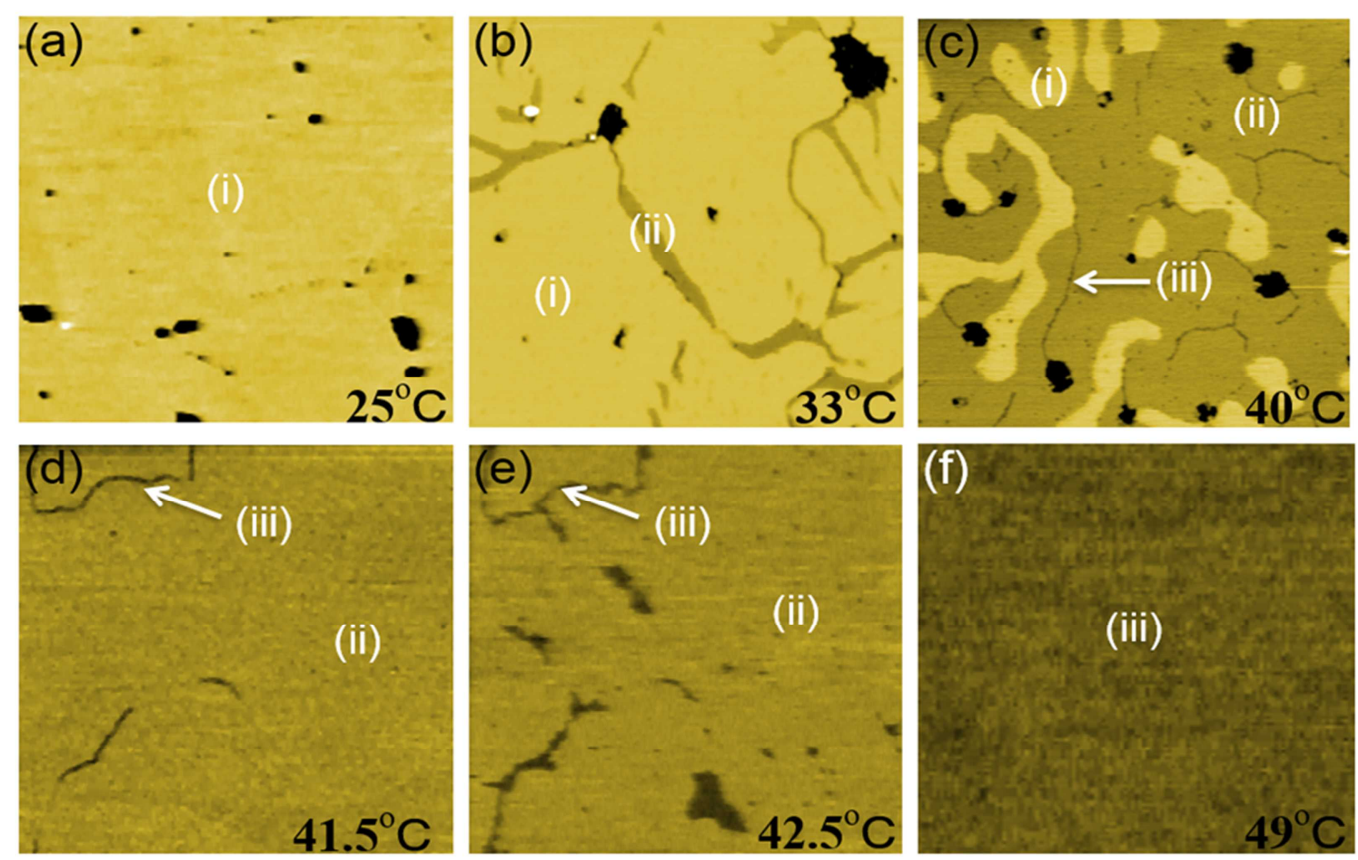

Figure 4. AFM images for a supported DPPC bilayer recorded at ${ }^{+}$(a) $25^{\circ} \mathrm{C}$, (b) $33^{\circ} \mathrm{C}$, (c) $40^{\circ} \mathrm{C}$, (d) $41.5^{\circ} \mathrm{C}$, (e) $42.5^{\circ} \mathrm{C}$, and (f) $49^{\circ} \mathrm{C}$ in a tris-buffer solution containing $5 \mathrm{mM} \mathrm{Ca}{ }^{2}$. Size of AFM images is $2 \mu \mathrm{m} \times 2 \mu \mathrm{m}$. The cross section profiles are given in Fig. S3. 
fact that the supported lipid bilayer sequentially melts with temperature over a wide temperature range as already discussed (Fig. 1b). ${ }^{35}$ Keller et al. have investigated the phase transition of the DPPC bilayer with $20 \%$ of 1-stearoyl-2-oleoyl-sn-phosphatidylcholine (SOPC) which can form granular bilayers with DPPC on a mica surface and observed the similar intermediate phase of domain (ii). ${ }^{34}$ They attributed phase (ii) to the melting of the top leaflet before the bottom leaflet is melted to form the full liquid phase of domain (iii) for the supported DPPC bilayer, especially occurring from the grain boundaries.

Therefore, we propose that the supported DPPC bilayer melts step-by-step in the following stages: (1) both leaflets are in the gel phase as domain (i); (2) the top leaflet starts to melt to form domain (ii); and (3) the bottom leaflet starts to melt to form domain (iii). These features are generally similar to recent AFM observations suggesting the "crack phase model" (Fig. 1b). ${ }^{33,35,41}$

It is interesting to note that pits and holes in the bilayer always appear on the supported DPPC bilayer surface in the gel phase (domain (i), see Fig. 4a-c). Similar phenomena have also been observed on the supported lipid bilayers prepared by either LB method or vesicle fusion method. ${ }^{30,33,35}$ It is noteworthy that these pits are observed only on the lipid bilayers in the gel-phase, but completely disappear in the liquid-phase when the temperature is higher than the $T_{m}$ (see, for example, Figs. $4 \mathrm{f}$ and $4 \mathrm{a}$ ). Although these phenomena of pits and holes are widely observed, no real agreement has been reached for their formation mechanism. In fact, we could not see any defect on the first monolayer deposited on the same substrate. We tentatively suggest that the second layer of the DPPC bilayer could be transferred as a fluid-like phase from the air/water interface to the first layer of DPPC in the downstroke dipping process but reconstructed into the gel phase which leaves more free space as pits and holes. Due to the lower coverage density of the phospholipids in the fluid-phase than that in gel-phase, pits can be formed in the bilayer. ${ }^{1}$

To further understand the melting mechanism of the supported DPPC bilayer, the 
populations of domains (ii) and (iii) at different temperatures were symmetrically evaluated from many independent AFM measurements. Figure 5a shows the fraction $(\alpha)$ of domain (ii) (black squares, $\left.\alpha_{(i i)}\right)$ and domain (iii) (red circles, $\left.\alpha_{(i i i)}\right)$ on the supported DPPC bilayer surface as a function of the temperature. The two melting stages of the bilayer can be clearly confirmed from the figure. $\alpha_{(i i)}$ appears from ca. $32^{\circ} \mathrm{C}$ and gradually increases with temperature. $\alpha_{(i i)}$ approaches $100 \%$ around $41^{\circ} \mathrm{C}$. At the same time, $\alpha_{(\text {(ii) }}$ starts to grow with temperature and finally reaches $100 \%$ around $48^{\circ} \mathrm{C}$. During the second melting stage, $\alpha_{(\text {ii) }}$ decreases with the increase of $\alpha_{(\text {iii })}$ while $\alpha_{(\text {ii) }}$ is considered as $\left(1-\alpha_{(\text {iii) }}\right)$ which was not shown in Fig.5a. With the increasing temperature, the supported DPPC bilayer in the gel phase (domain (i)) stepwise melts from the top leaflet (domain (ii)) to the bottom leaflet (domain (iii)) over a wide temperature range from $32^{\circ} \mathrm{C}$ to $48^{\circ} \mathrm{C}$. Domain (ii) can be considered as an important intermediate stage for the phase transition process. Next, we quantitatively analyze the phase transition of the supported DPPC bilayer in two stages, i.e., (1) melting of domains (i) to (ii), i.e., $\alpha_{(i i)}: 0 \rightarrow 100 \%$; (2) melting of domains (ii) to (iii), i.e., $\alpha$ (iii): $0 \rightarrow 100 \%$, by the van't Hoff equation. $34,39,41$

Assuming that the standard melting enthalpy $(\Delta \mathrm{H})$ is independent of the temperature, according to the van't Hoff equation, we have

$$
\frac{d(\ln K)}{d(1 / T)}=-\frac{\Delta \mathrm{H}}{R}
$$

where $K$ is the equilibrium constant, $\alpha /[(1-\beta)-\alpha] . \beta$ is a parameter correcting the pits on the bilayer surface. Both $\alpha$ and $\beta$ at temperature $T$ were determined by the AFM results shown in Fig. 5a. Figure $5 b$ shows $\ln K$ for domains (ii) and (iii) and plotted as a function of $1 / T$. Linear relationships between $\ln K$ and $1 / T$ are found for the two processes, i.e., melting of the topmost layer (domain (i) $\rightarrow$ (ii)) and bottom leaflet (domain (ii) $\rightarrow$ (iii)). The solid lines in Fig. $5 b$ are the fitted results based on the van't Hoff equation (1). From the slope, $\Delta H$ for the melting process of the top and bottom leaflets are estimated to be $300 \pm 38 \mathrm{~kJ} / \mathrm{mol}$ and 
$826 \pm 52 \mathrm{~kJ} / \mathrm{mol}$, respectively. A large discrepancy in $\Delta \mathrm{H}$ is observed for the melting top and bottom leaflets of the supported DPPC bilayer. $\triangle \mathrm{H}$ for the melting bottom leaflet is almost three times higher than that of melting top leaflet.

Keller et al. ${ }^{34}$ previously reported that the $\Delta \mathrm{H}$ for melting the bottom leaflet $(1036 \pm 26$ $\mathrm{kJ} / \mathrm{mol})$ is much higher than that of the top leaflet $(372 \pm 29 \mathrm{~kJ} / \mathrm{mol})$, which is comparable to that of the SUVs $(400 \mathrm{~kJ} / \mathrm{mol})$, but much higher than that of the LMVs $(35 \mathrm{~kJ} / \mathrm{mol})$. The high $\Delta \mathrm{H}$ for melting the bottom leaflet has been attributed to the effect of the strong interaction of the polar group of the DPPC with the solid substrate. Although some differences are still present between the present observation and previous AFM results, generally, good agreements have been achieved. Most of the previous AFM studies were about the lipid bilayers prepared by the vesicle fusion method, different from the LB method used in the study. This indicates that the sample preparation does not significantly affect the phase transition behaviors for the supported lipid bilayers. The interaction between the DPPC and substrate is expected to play an important role in the melting
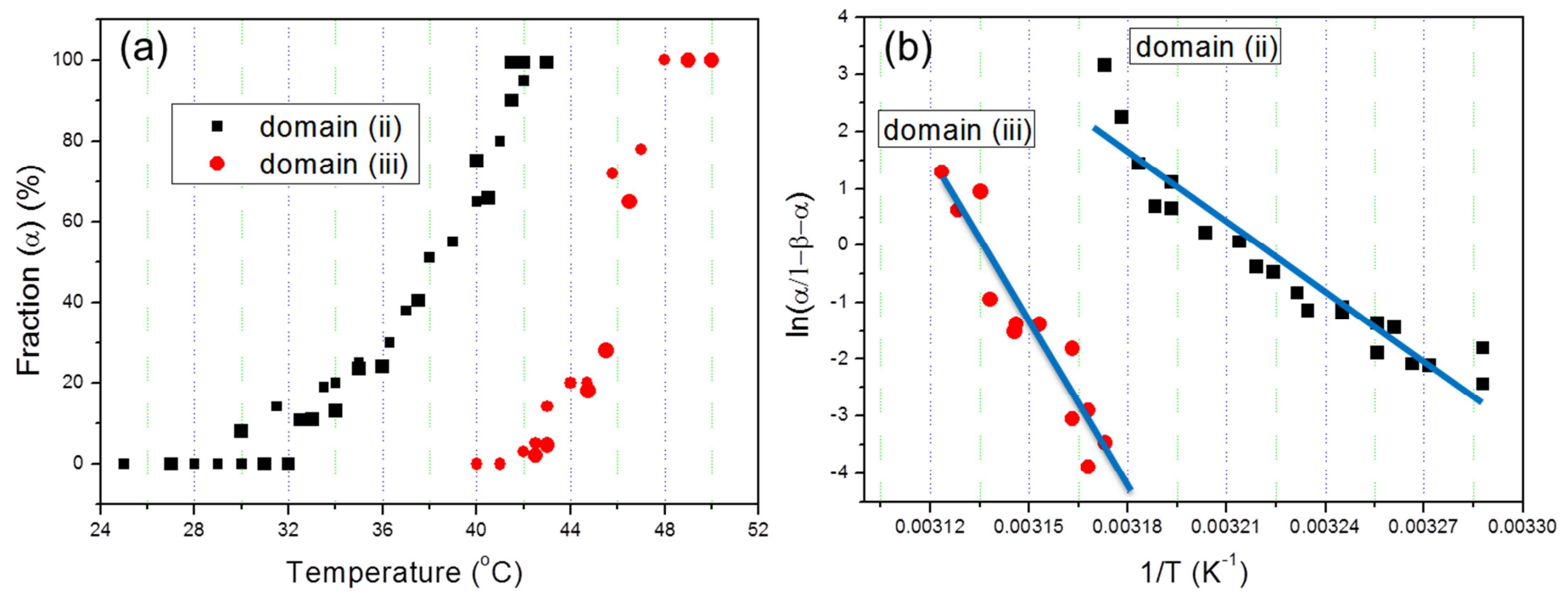

Figure 5. (a) Fraction of domain (ii) (black squares) and (iii) (red circles) determined from many in situ AFM observations as a function of temperature. (b) The van't Hoff plot for equilibrium constant as a function of $1 / T$. $T$ is the absolute temperature. The straight lines are fitted by van't Hoff equation. See text for details. 
process of the bottom leaflet.

On the other hand, Keller et al. proposed that the bottom leaflet does not begin to melt until the top monolayer is completely liquid and suggested that melting of the top layer is a prerequisite for the melting of the bottom monolayer. This is generally true for most of the bilayer surfaces based on the present AFM observations, but it was found that some limited regions show different features. As mentioned in Figs. 5c and 5d, domain (iii) already appears in addition to domains (i) and (ii). The coexistence of three phases, domains (i), (ii) and (iii), is exactly confirmed in the AFM image although the amount of domain (iii) is very low $(\sim 1 \%)$. There is controversy about the phase transition model mentioned above which only favors a layer by layer fashion.

\section{Comparison between SFG and AFM observations during the phase transition}

As already described, the structural changes in the supported DPPC bilayers during its phase transition process has been investigated by both SFG and AFM observations. The two independent measurements have provided comprehensive information to understand the mechanism of the phase transition process. From the definition for domains (i), (ii) and (iii) in the AFM observations (Fig. 4), it is easy to know that the fraction of domain (ii), $\alpha_{i i}$, should be the origin for the SFG signal from the symmetric bilayer of the DPPC. This fraction is directly associated with the asymmetry generated during the phase transition process of the symmetric bilayer. 
Figure 6 shows $\alpha_{i i}$ (filled circles) and $\left(I_{S F G}\right)^{1 / 2}$, square root of the SFG intensity at $2878 \mathrm{~cm}^{-1}$, as a function of temperature. The square root of the SFG intensity at $2878 \mathrm{~cm}^{-1}$ is used since it is proportional to the population of the target species (i.e., DPPC in domain (ii)) based on the theory of SFG. ${ }^{42-47}$ It is interesting to note that the SFG profile fits well with the fraction domain (ii), i.e., $\alpha_{\mathrm{ii}}$. Both measurements of the AFM and SFG exhibit a maximum around $41^{\circ} \mathrm{C}$, indicating that the two independent observations well correlate with each other. This confirmed that the SFG intensity directly depends on the fraction of domain (ii), which can generate the largest asymmetric feature, in the symmetric lipid bilayer (Fig. 1b). Although the two models proposed by Conboy et al. (Figs. 1c and 1d) ${ }^{27-28}$ could explain the observed SFG behaviors, these models does not fit with the surface structures determined by the AFM observations. The AFM observations showed that the top and bottom leaflets are

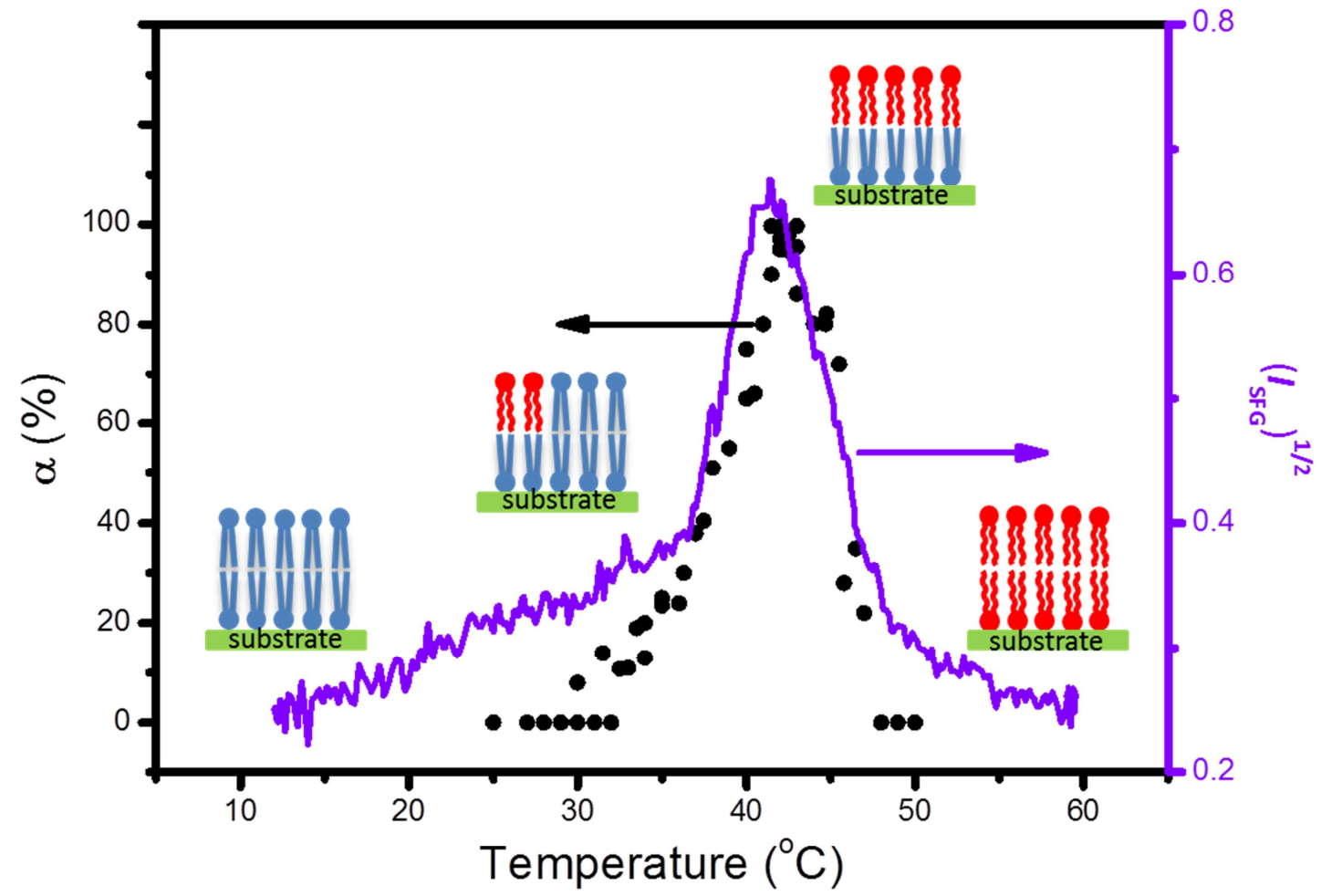

Figure 6. The fraction of domain (ii), $\alpha$ (filled circles) from in situ AFM observations and the square root of SFG intensities at $2878 \mathrm{~cm}^{-1}$ (solid trace) are plotted as a function of temperature. Schematic structural models for the supported bilayer are also given in the figure for each temperature region. See text for details. 
sequentially melted. This is also demonstrated by the higher melting enthalpy for the bottom layer than that of the top leaflet due to the interaction between the lipid and substrate.

On the other hand, as shown in Figs. $4 \mathrm{c}$ and $4 \mathrm{~d}$, a very small amount of the bottom layer $(<1 \%)$ is already melted (domain (iii)) before the top leaflet is fully melted. In fact, this situation is very similar to the domain size disparity model (Fig. 1d) proposed by Conboy et al..27-28 In other words, the domain size disparity model can also contribute to the phase transition process of the supported DPPC bilayer but in a very small ratio.

Based these discussions, we propose that a fraction of domain (ii) will contribute to the SFG intensity. Schematic structural models for the supported bilayer are given as insets in Fig. 6 in each temperature region. We think the present model (i.e., crack model in Fig. 1b) is the most reasonable model to explain the phase transition process of the supported lipid bilayer.

It is worthwhile to note that the different substrates and solutions can also induce some different behaviors during the phase transition process for the lipid bilayers. In the present study, our SFG observations were mainly carried out on fused quartz and $\mathrm{SiO}_{2}$-coated $\mathrm{CaF}_{2}$ surface (even bare $\mathrm{CaF}_{2}$ surface) while AFM observations were carried out on the mica surface due to the experimental reasons. Although substrate may partially affect the phase transition temperature of the lipid bilayers, it is hard to consider that this can fully change the phase transition mechanism. It is known that a water thin-layer is always present between the hydrophilic substrate and lipid layer from the sample preparation, lipid molecules are expected to interact with substrate through the water thin-layer instead of direct chemical interaction. This considerably weakens the influence from the substrates used. Furthermore, as mentioned in Experimental, our AFM observations did not find any differences between pure water and Tris-buffer solution. Figures S4-S5 shows the decoupled phase transition behavior of DPPC bilayer in a water solution. Previous studies 
have reported several influences of substrate and solution on the lipid bilayers. ${ }^{40-41} 56$ Most of these works were carried out using the unsaturated lipids which can show more complicated behaviors ${ }^{57}$ in comparison with the saturated lipid molecules used in the present study. Detailed experiments on the unsaturated lipid system are now in progress.

\section{Conclusions}

In the present study, the phase transition process of the supported DPPC bilayer has been systematically investigated by in situ SFG and AFM measurements. The SFG and AFM results showed very similar temperature-dependent profiles. The present study demonstrated that the top and bottom leaflets of the supported DPPC bilayer are sequentially melted over a wide temperature range during the phase transition process. The phase transition enthalpy for the bottom leaflet is much higher than that for the top one, indicating an interaction of the lipid molecules with the substrate. The SFG and AFM results showed very similar temperature-dependent profiles. The current study provided important information about the dynamic mechanism of the phase transition for the supported lipid bilayers which can mimic the biomembrane.

\section{Supporting Information}

Details about the in situ SFG observations for the cooling process from the liquid to gel phase of the asymmetric bilayer are given. The calculation for the tilt angle of the alkyl chain of the lipids before the phase transition is described. Cross section profiles for the AFM images as well as AFM images/ domain fractions determined pure water for the DPPC bilayers during heating process are also given. 


\section{Corresponding Author}

ye@cat.hokudai.ac.jp

\section{ACKNOWLEDGMENT}

This work was supported by a Grant-in-Aid for Scientific Research on Innovative Areas "Coordination Program" 759 (24108701) and a Grant-in-Aid for Scientific Research (B) 23350058 from the Ministry of Education, Culture, Sports, Science \& Technology (MEXT), Japan. L.N. acknowledges a scholarship from the China Scholarship Council (CSC).

\section{References}

1. Alberts, B.; Johnson, A.; Lewis, J.; Raff, M.; Roberts, K.; Walter, P., Molecular Biology of the Cell. 5th ed.; Garland Science: New York, 2008.

2. Simons, K.; Ikonen, E., Functional rafts in cell membranes, Nature 1997, 387, 569-572.

3. Sackmann, E., Supported Membranes: Scientific and Practical Applications Science 1996, 271, 43-48.

4. Janiak, M. J.; Small, D. M.; Shipley, G. G., Temperature and compositional dependence of the structure of hydrated dimyristoyl lecithin, J. Biol. Chem. 1979, 254, 6068-6078.

5. Heimburg, T., Thermal Biophysics of Membranes. Wiley-VCH Verlag GmbH \& Co. KGaA: Weinheim, 2007.

6. Pope, J. M.; Walker, L.; Cornell, B. A.; Francis, G. W., NMR study of synthetic lecithin bilayers in the vicinity of the gel-liquid--crystal transition, Biophys. J. 1981, 35, 509-520.

7. Brown, K. G.; Peticolos, W. L.; Brown, E., Raman studies of conformational changes in model membrane systems, Biochem. Biophys. Res. Commun. 1973, 54, 358-364.

8. Mantsch, H. H.; McElhaney, R. N., Phospholipid phase transitions in model and biological membranes as studied by infrared spectroscopy, Chem. Phys. Lipid 1991, 57, 213-226.

9. Cameron, D. G.; Casal, H. L.; Mantsch, H. H., Characterization of the pretransition in 1,2-dipalmitoyl-sn-glycero-3-phosphocholine by Fourier transform infrared spectroscopy, Biochemistry 1980, 19, 3665-3672.

10. Liu, J.; Conboy, J., Direct measurement of the transbilayer movement of phospholipids by sum-frequency vibrational spectroscopy, J. Am. Chem. Soc. 2004, 126, 8376-8377.

11. Sun, W. J.; Tristram-Nagle, S.; Suter, R. M.; Nagle, J. F., Structure of gel phase saturated lecithin bilayers: temperature and chain length dependence, Biophys. J. 1996, 71, 885-891.

12. Leidy, C.; Ocampo, J.; Duelund, L.; Mouritsen, Ole G.; Jørgensen, K.; Peters, Günther H., Membrane Restructuring by Phospholipase A2 Is Regulated by the Presence of Lipid Domains, Biophys. J. 2011, 101, 
90-99.

13. Moraille, P.; Badia, A., Enzymatic lithography of phospholipid bilayer films by stereoselective hydrolysis J. Am. Chem. Soc. 2005, 127, 6546-6547.

14. Gudmand, M.; Rocha, S.; Hatzakis, N. S.; Peneva, K.; Mullen, K.; Stamou, D.; Uji-I, H.; Hofkens, J.; Bjornholm, T.; Heimburg, T., Influence of lipid heterogeneity and phase behavior on phospholipase $\mathrm{A}_{2}$ action at the single molecule level, Biophys. J. 2010, 98, 1873-1882.

15. Sanchez, S. A.; Bagatolli, L. A.; Gratton, E.; Hazlett, T. L., A two-photon view of an enzyme at work: Crotalus atrox venom PLA(2) interaction with single-lipid and mixed-lipid giant unilamellar vesicles, Biophys. J. 2002, 82, 2232-2243.

16. Mabrey, S.; Sturtevant, J. M., Investigation of phase transitions of lipids and lipid mixtures by sensitivity differential scanning calorimetry, PNAS 1976, 73, 3862-3866.

17. Albon, N.; Sturtevant, J. M., Nature of the gel to liquid crystal transition of synthetic phosphatidylcholines, PNAS 1978, 75, 2258-2260.

18. Koynova, R.; Caffrey, M., Phases and phase transitions of the phosphatidylcholines, Biochim. Biophys. Acta 1998, 1376, 91-145.

19. Biltonen, R. L., A statistical-thermodynamic view of cooperative structural changes in phospholipid bilayer membranes: their potential role in biological function, J. Chem. Thermodynamics 1990, 22, 1-19.

20. Nagano, H.; Nakanishi, T.; Yao, H.; Ema, K., Effect of vesicle size on the heat capacity anomaly at the gel to liquid-crystalline phase transition in unilamellar vesicles of dimyristoylphosphatidylcholine, Phys. Rev. E $1995,52,4244-4250$.

21. El Kirat, K.; Morandat, S.; Dufrene, Y. F., Nanoscale analysis of supported lipid bilayers using atomic force microscopy, Biochim. Biophys. Acta 2010, 1798, 750-765.

22. Giocondi, M. C.; Yamamoto, D.; Lesniewska, E.; Milhiet, P. E.; Ando, T.; Le Grimellec, C., Surface topography of membrane domains, Biochim. Biophys. Acta 2010, 1798, 703-718.

23. Yang, J.; Appleyard, J., The main phase transition of mica-supported phosphatidylcholine membranes, J. Phys. Chem. B 2000, 104, 8097-8100.

24. Lee, C. S.; Bain, C. D., Raman spectra of planar supported lipid bilayers, Biochim. Biophys. Acta 2005, 1711, 59-71.

25. Tamm, L. K.; Tatulian, S. A., Infrared spectroscopy of proteins and peptides in lipid bilayers, Quarterly Reviews of Biophysics 1997, 30, 365-429.

26. Liu, J.; Conboy, J. C., Phase transition of a single lipid bilayer measured by sum-frequency vibrational spectroscopy, J. Am. Chem. Soc. 2004, 126, 8894-8895.

27. Liu, J.; Conboy, J., Asymmetric Distribution of Lipids in a Phase Segregated Phospholipid Bilayer Observed by Sum-Frequency Vibrational Spectroscopy, J. Phys. Chem. C 2007, 111 (25), 8988-8999.

28. Liu, J.; Conboy, J., Structure of a Gel Phase Lipid Bilayer Prepared by the Langmuir-Blodgett/Langmuir-Schaefer Method Characterized by Sum-Frequency Vibrational Spectroscopy Langmuir 2005, 21, 9091-9097.

29. Tong, Y.; Li, N.; Liu, H.; Ge, A.; Osawa, M.; Ye, S., Mechanistic studies by sum-frequency generation 
spectroscopy: Hydrolysis of a supported phospholipid bilayer by phospholipase $\mathrm{A}_{2}$, Angew. Chem. Int. Ed. 2010, 49, 2319-2323.

30. Leonenko, Z. V.; Finot, E.; Ma, H.; Dahms, T. E. S.; Cramb, D. T., Investigation of temperature-induced phase transitions in DOPC and DPPC phospholipid bilayers using temperature-controlled scanning force microscopy, Biophys. J. 2004, 86, 3783-3793.

31. Schuy, S.; Janshoff, A., Thermal Expansion of Microstructured DMPC Bilayers Quantified by Temperature-Controlled Atomic Force Microscopy, ChemPhysChem 2006, 7 (6), 1207-1210.

32. Charrier, A.; Thibaudau, F., Main phase transitions in supported lipid single-bilayer, Biophys. J. 2005, 89, 1094-1101.

33. Garcia-Manyes, S.; Oncins, G.; Sanz, F., Effect of Temperature on the Nanomechanics of Lipid Bilayers Studied by Force Spectroscopy, Biophys. J. 2005, 89, 4261-4274.

34. Keller, D.; Larsen, N. B.; Moller, I. M.; Mouritsen, O. G., Decoupled phase transitions and grain-boundary melting in supported phospholipid bilayers, Phys. Rev. Lett. 2005, 94, 025701

35. Feng, Z. V.; Spurlin, T. A.; Gewirth, A. A., Direct visualization of asymmetric behavior in supported lipid bilayers at the gel-fluid phase transition, Biophys. J. 2005, 88, 2154-2164.

36. Wu, H.; Yu, L.; Tong, Y.; Ge, A.; Yau, S.; Osawa, M.; Ye, S., Enzyme-catalyzed hydrolysis of the supported phospholipid bilayers studied by atomic force microscopy, Biochim. Biophys. Acta 2013, 1828, 642-651.

37. Tokumasu, F.; Jin, A. J.; Dvorak, J. A., Lipid membrane phase behaviour elucidated in real time by controlled environment atomic force microscopy, Journal of Electron Microscopy 2002, 51, 1-9.

38. Tokumasu, F.; Jin, A. J.; Feigenson, G. W.; Dvorak, J. A., Atomic force microscopy of nanometric liposome adsorption and nanoscopic membrane domain formation, Ultramicroscopy 2003, 97, 217-227.

39. Xie, A. F.; Yamada, R.; Gewirth, A. A.; Granick, S., Materials science of the gel to fluid phase transition in a supported phospholipid bilayer, Phys. Rev. Lett. 2002, 89, 246103.

40. Seeger, H. M.; Cerbo, A. D.; Alessandrini, A.; Facci, P., Supported Lipid Bilayers on Mica and Silicon Oxide: Comparison of the Main Phase Transition Behavior, J. Phys. Chem. B 2010, 114, 8926-8933.

41. Seeger, H. M.; Marino, G.; Alessandrini, A.; Facci, P., Effect of Physical Parameters on the Main Phase Transition of Supported Lipid Bilayers, Biophys. J. 2009, 97, 1067-1076.

42. Shen, Y. R., The Principles of Nonlinear Optics. John Wiley \& Sons, Inc.: New York, 1984.

43. Shen, Y. R., Phase-Sensitive Sum-Frequency Spectroscopy, Annu. Rev. Phys. Chem. 2013, 64, 129-150.

44. Richmond, G. L., Molecular Bonding and Interactions at Aqueous Surfaces as Probed by Vibrational Sum Frequency Spectroscopy, Chem. Rev. 2002, 102, 2693-2724.

45. Chen, Z.; Shen, Y. R.; Somorjai, G. A., Studies of Polymer Surfaces by Sum Frequency Generation Vibrational Spectroscopy, Annu. Rev. Phys. Chem. 2002, 53, 437-465.

46. Holman, J.; Davies, P. B.; Nishida, T.; Ye, S.; Neivandt, D. J., Sum frequency generation from Langmuir-Blodgett multilayer films on metal and dielectric substrates, J. Phys. Chem. B (Feature Article) 2005, 109, $18723-18732$.

47. Ye, S.; Tong, Y.; Ge, A.; Qiao, L.; Davies, P. B., Interfacial Structure of Soft Matter Probed by SFG 
Spectroscopy, The Chemical Record 2014, 14, 791-805.

48. Ye, S.; Noda, H.; Morita, S.; Uosaki, K.; Osawa, M., Surface molecular structures of Langmuir-Blodgett films of stearic acid on the solid substrate studied by sum frequency generation spectroscopy, Langmuir 2003, $19,2238-2242$.

49. Ye, S.; Noda, H.; Nishida, T.; Morita, S.; Osawa, M., $\mathrm{Cd}^{2+}$-induced interfacial structural changes of Langmuir-Blodgett films of stearic acid on solid substrates: A sum frequency generation study, Langmuir 2004, 20, 357-365.

50. Ge, A.; Wu, H.; Darwish, T. A.; James, M.; Osawa, M.; Ye, S., Structure and Lateral Interaction in Mixed Monolayers of Dioctadecyldimethylammonium Chloride (DOAC) and Stearyl Alcohol, Langmuir 2013, 29, 5407-5417.

51. Ge, A.; Peng, Q.; Wu, H.; Liu, H.; Tong, Y.; Nishida, T.; Yoshida, N.; Suzuki, K.; Sakai, T.; Osawa, M.; Ye, S., Effect of Functional Group on the Monolayer Structures of Biodegradable Quaternary Ammonium Surfactants, Langmuir 2013, 29, 14411-14420.

52. Conboy, J. C.; Messmer, M. C.; Richmond, G. L., Dependence of Alkyl Chain Conformation of Simple Ionic Surfactants on Head Group Functionality As Studied by Vibrational Sum-Frequency Spectroscopy, J. Phys. Chem. B. 1997, 101, 6724-6733.

53. Briggs, A. M.; Johal, M. S.; Davies, P. B.; Cooke, D. J., Structure and thermal stability of dichain sugar surfactants at the solid/water interface studied by sum-frequency vibrational spectroscopy, Langmuir 1999, 15, 1817-1828.

54. Grandbois, M.; Clausen-Schaumann, H.; Gaub, H., Atomic force microscope Imaging of phospholipid bilayer degradation by phospholipase A 2 Biophys. J. 1998, 74, 2398-2404.

55. Lis, L. J.; McAlister, M.; Fuller, N.; Rand, R. P.; Parsegian, V. A., Interactions between neutral phospholipid bilayer membranes, Biophys. J. 1982, 37, 657-665.

56. Cremer, P. S.; Boxer, S. G., Formation and Spreading of Lipid Bilayers on Planar Glass Supports, J. Phys. Chem. B 1999, 103, 2554-2559.

57. Qiao, L.; Ge, A.; Osawa, M.; Ye, S., Structure and stability studies of mixed monolayers of saturated and unsaturated phospholipids under low-level ozone, Phys. Chem. Chem. Phys. 2013, 15, 17775-17785. 
TOC

Phase Transition for the Supported Lipid Bilayer

$\frac{\text { Low }}{\text { Temperature }}$ 